\title{
THE FIBERING MAP APPROACH FOR A SINGULAR ELLIPTIC SYSTEM INVOLVING THE $p(x)$-LAPLACIAN AND NONLINEAR BOUNDARY CONDITIONS
}

\author{
MOUNA KRATOU AND KAMEL SAOUDI
}

ABStRaCt. The purpose of this work is to study the existence and multiplicity of positive solutions for a class of singular elliptic systems involving the $p(x)$ Laplace operator and nonlinear boundary conditions.

\section{INTRODUCTION}

This paper is concerned with the multiplicity of positive solutions for the following singular elliptic system involving the $p(x)$-Laplace operator and sub-linear Neumann nonlinearities:

$$
\left\{\begin{array}{l}
-\Delta_{p(x)} u+|u|^{p(x)-2} u(x)=\lambda a(x)|u|^{-\alpha(x)} \quad \text { in } \Omega \\
-\Delta_{p(x)} v+|v|^{p(x)-2} v(x)=\mu b(x)|v|^{-\alpha(x)} \quad \text { in } \Omega, \\
|\nabla u|^{p(x)-2} \frac{\partial u}{\partial \nu}=c(x) \frac{q(x)}{q(x)+r(x)} u^{q(x)-2} u|v|^{r(x)} \quad \text { on } \partial \Omega \\
|\nabla v|^{p(x)-2} \frac{\partial v}{\partial \nu}=c(x) \frac{r(x)}{q(x)+r(x)} u^{q(x)}|v|^{r(x)-2} v \quad \text { on } \partial \Omega .
\end{array}\right.
$$

Here $\Omega \subset \mathbb{R}^{N}(N \geq 2)$ is a bounded domain with $C^{2}$ boundary; $\lambda, \mu$ are two parameters; $a, b, c \in C(\bar{\Omega})$ are non-negative weight functions with compact support in $\Omega$. For any continuous and bounded function $a$ we define $a^{+}:=\operatorname{ess} \sup a(x)$ and $a^{-}:=\operatorname{ess} \inf a(x)$. We assume the following on $p, q, r$ and $\alpha$ :

(A0) $\alpha(x) \in C(\bar{\Omega})$ satisfies $0<\alpha^{-} \leq \alpha(x) \leq \alpha^{+}<1$.

(A1) $p(x), q(x), r(x) \in C(\bar{\Omega})$ are such that $0<1-\alpha(x)<p(x)<q(x)+r(x)<$ $p^{*}(x)$ (where $p^{*}(x)=\frac{N p(x)}{N-p(x)}$ if $p(x)<N$ and $p^{*}(x)=\infty$ if $p(x) \geq N$ ), and $p^{-} \leq p^{+}<q^{-}+r^{-} \leq q^{+}+r^{+}$.

2020 Mathematics Subject Classification. 35J20, 35J60, 35J70, 47J10, 46E35.

Key words and phrases. Fibering map; singular system; Neumann boundary conditions; $p(x)$ Laplace operator; generalized Lebesgue Sobolev spaces.

This work is supported by the Basic and Applied Scientific Research Center, Scientific Research Deanship, Imam Abdulrahman Bin Faisal University, KSA, under Award No. 2020-852-BASRC. 
(A2) $a(x), b(x), c(x) \geq 0, a(x) \in L^{r_{1}(x)}, b(x) \in L^{r_{2}(x)}, c(x) \in L^{r_{3}(x)}, r_{i} \in C(\bar{\Omega})$ $(i=1,2,3)$, where

$$
\begin{array}{r}
\frac{1}{r_{1}(x)}+\frac{1}{p^{*}(x) /(1-\alpha(x))}=1, \\
\frac{1}{r_{2}(x)}+\frac{1}{p^{*}(x) /(1-\alpha(x))}=1, \\
\frac{1}{r_{3}(x)}+\frac{1}{p^{*}(x) / q(x)}+\frac{1}{p^{*}(x) / r(x)}=1 .
\end{array}
$$

The operator $\Delta_{p(x)} u:=\operatorname{div}\left(|\nabla u|^{p(x)-2} \nabla u\right)$, where $p$ is a continuous non-constant function, is called $p(x)$-Laplace. This differential operator is a natural generalization of the $p$-Laplace operator $\Delta_{p} u:=\operatorname{div}\left(|\nabla u|^{p-2} \nabla u\right)$, where $p>1$ is a real constant. However, the $p(x)$-Laplace operator possesses more complicated nonlinearity than the $p$-Laplace operator, due to the fact that $\Delta_{p(x)}$ is not homogeneous. This fact implies some difficulties; for example, we can not use the Lagrange multiplier theorem in many problems involving this operator.

The study of differential and partial differential equations involving variable exponent is a new topic. The interest in studying such problems was stimulated by their applications in elastic mechanics, fluid dynamics, electrorheological fluids, image processing, flow in porous media, calculus of variations, non-linear elasticity theory, heterogeneous porous media models, etc. (see [1, 5]). These physical problems were facilitated by the development of Lebesgue and Sobolev spaces with variable exponent.

Before giving our main results, let us briefly recall literature concerning related non-linear equations involving the $p(x)$-Laplace operator. The existence and multiplicity of solutions of elliptic equations with variable exponents involving the $p(x)$-Laplace operator have been extensively investigated using various methods, specially variational techniques, and have received much attention. In that context, we would like to mention [2, 9, 10, 17, 19, 25, 26, 27, 29] and the references therein.

The fibering map approach for describing the Nehari manifolds and seeking solutions in an appropriate subset of the Sobolev space is introduced by Drabek and Pohozaev in [6]. In variable exponent cases this method has some difficulties in comparison with the Nehari manifolds approach in $p$-Laplacian problems. This is due to the non-homogeneity of the variable exponent $p$. Nevertheless, in recent years, several authors have used the Nehari manifold and fibering maps to solve quasilinear problems with variable exponent (see [18, 22, 30]).

Problem (1.1) has been also studied with different elliptic operators. We refer the reader to the monograph by Ghergu and Rădulescu [14] for a more general presentation of these results and the survey article of Crandall, Rabinowitz, and Tartar [4. After this, many authors have considered the problem above for Laplacian operators, $p$-Laplacian operators, fractional Laplacian or fractional $p$-Laplacian, using the technique used in [4] or a combination of this approach with Nehari's and Perron's methods; we would like to mention [3, 12, 13, 15, 24, 28. 
However, as far as we know, there are few results on $p(x)$-Laplacian systems with concave/convex nonlinearities (see [21, 23] and references therein). Motivated by the above results, in the present paper we are interested in the multiplicity of solutions for the singular $p(x)$-Laplacian system 1.1 by using the Nehari manifold decomposition.

Here we state our main result.

Theorem 1.1. Assume that (A0)-(A2) hold. Then, there exists a number $\Lambda_{0}>0$ defined by

$$
\Lambda_{0}:=\frac{c_{8}}{c_{9}}\left(\frac{p^{+}+\alpha^{+}-1}{q^{-}+r^{+}+\alpha^{+}-1}\right)^{\frac{p^{+}+\alpha^{+}-1}{q^{-}+r^{+}-p^{+}}}\left(\frac{p^{+}-q^{-}-r^{+}}{q^{-}+r^{+}+\alpha^{+}-1}\right),
$$

where $c_{8}, c_{9}$ are positive constants, such that the problem 1.1 has at least two non-negative solutions for all $0<\lambda+\mu<\Lambda_{0}$.

This paper is organized as follows. In Section 2, we will recall some basic facts about the variable exponent Lebesgue and Sobolev spaces which we will use later. In Section 3 we analyze the fibering map related to the Euler functional associated to the problem (1.1). Proofs of our results will be presented in Sections 4 and 5 .

\section{Generalized Lebesgue-Sobolev spaces Setting}

To deal with the $p(x)$-Laplacian problem, we need to introduce some functional spaces $L^{p(\cdot)}(\Omega), W^{1, p(\cdot)}(\Omega), W_{0}^{1, p(\cdot)}(\Omega)$, and some properties of the $p(x)$-Laplacian that we will use later. Denote by $S(\Omega)$ the set of all measurable real-valued functions defined in $\Omega$. Note that two measurable functions are considered as the same element of $S(\Omega)$ when they are equal almost everywhere. Let

$$
L^{p(\cdot)}(\Omega)=\left\{u \in S(\Omega): \int_{\Omega}|u(x)|^{p(x)} \mathrm{d} x<\infty\right\},
$$

with the norm

$$
|u|_{p(\cdot)}=|u|_{L^{p(\cdot)}(\Omega)}=\inf \left\{\lambda>0: \int_{\Omega}\left|\frac{u(x)}{\lambda}\right|^{p(x)} \mathrm{d} x \leq 1\right\}
$$

and

$$
L_{c(x)}^{p(\cdot)}(\Omega)=\left\{u \in S(\Omega): \int_{\Omega} c(x)|u(x)|^{p(x)} \mathrm{d} x<\infty\right\},
$$

where $c$ is a measurable real-valued function and $c(x)>0$ for $x \in \Omega$. The space $\left(L^{p(\cdot)}(\Omega),|\cdot|_{p(\cdot)}\right)$ becomes a Banach space. We call it variable exponent Lebesgue space. Moreover, this space is a separable, reflexive, and uniform convex Banach space; see [11, Theorems 1.6, 1.10, 1.14].

The variable exponent Sobolev space

$$
W^{1, p(\cdot)}(\Omega)=\left\{u \in L^{p(\cdot)}(\Omega):|\nabla u| \in L^{p(\cdot)}(\Omega)\right\}
$$

can be equipped with the norm

$$
\|u\|=|u|_{p(\cdot)}+|\nabla u|_{p(\cdot)}, \quad \text { for all } u \in W^{1, p(\cdot)}(\Omega) .
$$


Note that $W_{0}^{1, p(\cdot)}(\Omega)$ is the closure of $C_{0}^{\infty}(\Omega)$ in $W^{1, p(\cdot)}(\Omega)$ under the norm $\|u\|=$ $|\nabla u|_{p(\cdot)}$. The spaces $W^{1, p(\cdot)}(\Omega)$ and $W_{0}^{1, p(\cdot)}(\Omega)$ are separable, reflexive, and uniform convex Banach spaces (see [11, Theorem 2.1]). The inclusion between Lebesgue spaces also generalizes naturally: if $0<|\Omega|<\infty$ and $p_{1}, p_{2}$ are variable exponents so that $p_{1}(x) \leq p_{2}(x)$ almost everywhere in $\Omega$, then there exists the continuous embedding $L^{p_{2}(x)}(\Omega) \hookrightarrow L^{p_{1}(x)}(\Omega)$.

We denote by $L^{q(x)}(\Omega)$ the conjugate space of $L^{p(x)}(\Omega)$, where $\frac{1}{q(x)}+\frac{1}{p(x)}=1$. For $u \in L^{p(x)}(\Omega)$ and $v \in L^{q(x)}(\Omega)$, the Hölder type inequality

$$
\left|\int_{\Omega} u(x) v(x) \mathrm{d} x\right| \leq\left(\frac{1}{p^{-}}+\frac{1}{q^{-}}\right)|u|_{p(x)}|v|_{q(x)}
$$

holds true.

An important role in manipulating the generalized Lebesgue spaces is played by the modular of the $L^{p(x)}(\Omega)$ space, which is the mapping $\rho_{p(x)}: L^{p(x)}(\Omega) \rightarrow \mathbb{R}$ defined by

$$
\rho_{p(x)}(u)=\int_{\Omega}|u|^{p(x)} \mathrm{d} x .
$$

Lemma 2.1. If $\left(u_{n}\right), u \in L^{p(x)}(\Omega)$, and $p^{+}<\infty$, then the following relations hold true:

$$
\begin{aligned}
& \|u\|_{L^{p(x)}}>1 \Rightarrow\|u\|_{L^{p(x)}}^{p^{-}} \leq \rho_{p(x)}(u) \leq\|u\|_{L^{p(x)}}^{p^{+}}, \\
& \|u\|_{L^{p(x)}}<1 \Rightarrow\|u\|_{L^{p(x)}}^{p^{+}} \leq \rho_{p(x)}(u) \leq\|u\|_{L^{p(x)}}^{p^{-}}, \\
& \left\|u_{n}-u\right\|_{L^{p(x)}} \rightarrow 0 \text { if and only if } \rho_{p(x)}\left(u_{n}-u\right) \rightarrow 0 .
\end{aligned}
$$

The following result generalizes the well-known Sobolev embedding theorem.

Theorem 2.2 (See [8, 16]). Let $\Omega \subset \mathbb{R}^{N}$ be an open bounded domain with Lipschitz boundary and assume that $p \in C(\bar{\Omega})$ with $p(x)>1$ for each $x \in \bar{\Omega}$. If $r \in C(\bar{\Omega})$ and $p(x) \leq r(x) \leq p^{*}(x)$ for all $x \in \bar{\Omega}$, then there exists a continuous embedding $W^{1, p(x)}(\Omega) \hookrightarrow L^{r(x)}(\Omega)$. Also, the embedding is compact when $r(x)<p^{*}(x)$ almost everywhere in $\bar{\Omega}$, where

$$
p^{*}(x)= \begin{cases}\frac{N p(x)}{N-p(x)}, & \text { if } p(x)<N \\ +\infty, & \text { if } p(x) \geq N .\end{cases}
$$

The following three theorems play an important role in the present paper.

Theorem 2.3 (See [7]). If $|u|^{q(x)} \in L^{\frac{r(x)}{q(x)}}(\Omega)$, where $r(x), q(x) \in L_{+}^{\infty}(\Omega)$, such that $q(x) \leq r(x)$, then $u \in L^{r(x)}(\Omega)$ and there is a number $q_{0} \in\left[q^{-}, q^{+}\right]$such that $\left.\left.|| u\right|^{q(x)}\right|_{\frac{r(x)}{q(x)}}=\left(|u|_{r(x)}\right)^{q_{0}}$. 
Theorem 2.4 (See [7]). If $\frac{1}{p(x)}+\frac{1}{p^{\prime}(x)}+\frac{1}{p^{\prime \prime}(x)}=1$, then for any $u \in L^{p(x)}(\Omega)$, $v \in L^{p^{\prime}(x)}(\Omega)$, and $w \in L^{p^{\prime \prime}(x)}(\Omega)$, one has

$$
\begin{aligned}
\left|\int_{\Omega} u(x) v(x) w(x) \mathrm{d} x\right| & \leq\left(\frac{1}{p^{-}}+\frac{1}{p^{\prime-}}+\frac{1}{p^{\prime \prime-}}\right)|u|_{p(x)}|v|_{p^{\prime}(x)}|u|_{p^{\prime \prime}(x)} \\
& \leq 3|u|_{p(x)}|v|_{p^{\prime}(x)}|u|_{p^{\prime \prime}(x)} .
\end{aligned}
$$

Theorem 2.5 (See [20]). Assume that the boundary of $\Omega$ possesses the cone property and $p \in C(\bar{\Omega})$. Suppose that $b \in L^{\gamma(x)}, c(x)>0$ for $x \in \Omega, \gamma \in C(\bar{\Omega})$, and $\gamma^{-}>1, \gamma_{0}^{-} \leq \gamma_{0}(x) \leq \gamma_{0}^{+}\left(\frac{1}{\gamma(x)}+\frac{1}{\gamma_{0}(x)}=1\right)$. If $q \in C(\bar{\Omega})$ and

or

$$
1<q(x)<\frac{\gamma(x)-1}{\gamma(x)} p_{\partial}^{*}(x), \quad \text { for all } x \in \bar{\Omega},
$$

$$
1<\gamma(x)<\frac{N \gamma(x)}{N \gamma(x)-r(x)(N-p(x))},
$$

then the embedding from $W^{1, p(x)}(\Omega)$ to $L_{c(x)}^{q(x)}(\partial \Omega)$ is compact.

\section{FibERING MAP ANALYSIS FOR PROBLEM 1.1}

In what follows, $W$ will denote the Cartesian product of two Sobolev spaces $W^{1, p(x)}(\Omega)$, i.e., $W=W^{1, p(x)}(\Omega) \times W^{1, p(x)}(\Omega)$. Let us choose on $W$ the norm $\|\cdot\|$ defined by

$$
\|(u, v)\|=\left(\int_{\Omega}\left(|\nabla u|^{p(x)}+|u|^{p(x)}\right) \mathrm{d} x+\int_{\Omega}\left(|\nabla v|^{p(x)}+|v|^{p(x)}\right) \mathrm{d} x\right)^{\frac{1}{p(x)}},
$$

which is equivalent to the standard one. by

Associated to the problem (1.1) we define the functional $E_{\lambda, \mu}: W \rightarrow \mathbb{R}$ given

$$
\begin{aligned}
E_{\lambda, \mu}(u, v):= & \int_{\Omega}\left(\frac{|\nabla u|^{p(x)}}{p(x)}+\frac{|u|^{p(x)}}{p(x)}\right) \mathrm{d} x+\int_{\Omega}\left(\frac{|\nabla v|^{p(x)}}{p(x)}+\frac{|v|^{p(x)}}{p(x)}\right) \mathrm{d} x \\
& -\int_{\Omega}\left(\frac{\lambda a(x)}{1-\alpha(x)}\left(u^{+}\right)^{1-\alpha(x)}+\frac{\mu b(x)}{1-\alpha(x)}\left(v^{+}\right)^{1-\alpha(x)}\right) \mathrm{d} x \\
& -\int_{\partial \Omega} \frac{c(x)}{q(x)+r(x)}|u|^{q(x)}|v|^{r(x)} \mathrm{d} x .
\end{aligned}
$$

Let

$$
\begin{aligned}
& L(u, v)=\int_{\Omega}\left(\frac{|\nabla u|^{p(x)}}{p(x)}+\frac{|u|^{p(x)}}{p(x)}\right) \mathrm{d} x+\int_{\Omega}\left(\frac{|\nabla v|^{p(x)}}{p(x)}+\frac{|v|^{p(x)}}{p(x)}\right) \mathrm{d} x, \\
& P(u, v)=\int_{\Omega}\left(\frac{\lambda a(x)}{1-\alpha(x)}\left(u^{+}\right)^{1-\alpha(x)}+\frac{\mu b(x)}{1-\alpha(x)}\left(v^{+}\right)^{1-\alpha(x)}\right) \mathrm{d} x
\end{aligned}
$$

and

$$
Q(u, v)=\int_{\partial \Omega} \frac{c(x)}{q(x)+r(x)}|u|^{q(x)}|v|^{r(x)} \mathrm{d} x .
$$


Note that using a new cut-off functional (see [29, Lemma A.3]) allows us to apply the variational method. Precisely, we obtain the $C^{1}$-differentiability of the associated cut-off functional.

Definition 3.1. We say that $(u, v) \in W$ is a weak solution of the problem $[1.1$ if we have

$$
\begin{aligned}
& \int_{\Omega}\left(|\nabla u|^{p(x)-2} \nabla u \nabla \phi+|u|^{p(x)-2} u \phi\right) \mathrm{d} x \\
& \quad=\lambda \int_{\Omega} a(x)\left(u^{+}\right)^{-\alpha(x)} \phi \mathrm{d} x+\int_{\partial \Omega} c(x) \frac{q(x)}{q(x)+r(x)}|u|^{q(x)-2} u|v|^{r(x)} \phi \mathrm{d} x,
\end{aligned}
$$

and

$$
\begin{aligned}
& \int_{\Omega}\left(|\nabla v|^{p(x)-2} \nabla v \nabla \psi+|v|^{p(x)-2} v \psi\right) \mathrm{d} x \\
& \quad=\mu \int_{\Omega} b(x)\left(v^{+}\right)^{-\alpha(x)} \psi \mathrm{d} x+\int_{\partial \Omega} c(x) \frac{r(x)}{q(x)+r(x)}|u|^{q(x)}|v|^{r(x)-2} v \psi \mathrm{d} x,
\end{aligned}
$$

for all $(\phi, \psi) \in C_{c}^{\infty}(\Omega) \times C_{c}^{\infty}(\Omega)$, where $C_{c}^{\infty}(\Omega)$ denotes the space of all $C^{\infty}$ functions with compact support in $\Omega$.

In many problems, such as problem (1.1), $E_{\lambda, \mu}$ is not bounded below on the whole space $W$, but is bounded below on the corresponding Nehari manifold, which is defined by

$$
\mathcal{N}_{\lambda, \mu}:=\left\{(u, v) \in W \backslash\{(0,0)\}:\left\langle E_{\lambda}^{\prime}(u, v),(u, v)\right\rangle=0\right\} .
$$

It is clear that all critical points of $E_{\lambda, \mu}$ must lie on $\mathcal{N}_{\lambda, \mu}$. We will see below that local minimizers of $E_{\lambda, \mu}$ on $\mathcal{N}_{\lambda, \mu}$ are usually critical points of $E_{\lambda, \mu}$. Then, it is easy to see that $u \in \mathcal{N}_{\lambda}$ if and only if

$$
\begin{aligned}
I_{\lambda, \mu}(u, v):= & \left\langle E_{\lambda, \mu}^{\prime}(u, v),(u, v)\right\rangle \\
= & \int_{\Omega}\left(|\nabla u|^{p(x)}+|u|^{p(x)}\right) \mathrm{d} x+\int_{\Omega}\left(|\nabla v|^{q(x)}+|v|^{q(x)}\right) \mathrm{d} x \\
& -\lambda \int_{\Omega} a(x)|u|^{1-\alpha(x)} \mathrm{d} x-\mu \int_{\Omega} b(x)|v|^{1-\alpha(x)} \mathrm{d} x-\int_{\partial \Omega} c(x)|u|^{q(x)}|v|^{q(x)} \mathrm{d} x \\
= & 0 .
\end{aligned}
$$

We note that $\mathcal{N}_{\lambda, \mu}$ contains every solution of problem $(1.1)$. Then, for $(u, v) \in \mathcal{N}_{\lambda, \mu}$ we have

$$
\begin{aligned}
\left\langle I_{\lambda, \mu}^{\prime}(u, v),(u, v)\right\rangle= & \int_{\Omega} p(x)\left(|\nabla u|^{p(x)}+|u|^{p(x)}\right) \mathrm{d} x+\int_{\Omega} p(x)\left(|\nabla v|^{p(x)}+|v|^{p(x)}\right) \mathrm{d} x \\
& -\int_{\Omega}(1-\alpha(x))\left(\lambda a(x)|u|^{1-\alpha(x)} \mathrm{d} x+\mu b(x)|v|^{1-\alpha(x)}\right) \mathrm{d} x \\
& -\int_{\partial \Omega} c(x)(q(x)+r(x))|u|^{q(x)}|v|^{r(x)} \mathrm{d} x .
\end{aligned}
$$


Now, we know that the Nehari manifold is closely related to the behavior of the functions $\Phi_{u, v}: t \mapsto E_{\lambda, \mu}(t u, t v)$ for $t>0$ defined by

$$
\begin{aligned}
\Phi_{u, v}(t)= & \int_{\Omega} \frac{t^{p(x)}}{p(x)}\left(|\nabla u|^{p(x)}+|u|^{p(x)}\right) \mathrm{d} x+\int_{\Omega} \frac{t^{p(x)}}{p(x)}\left(|\nabla v|^{p(x)}+|v|^{p(x)}\right) \mathrm{d} x \\
& -\int_{\Omega} \frac{t^{1-\alpha(x)}}{1-\alpha(x)}\left(\lambda a(x)|u|^{1-\alpha(x)}+\mu b(x)|v|^{1-\alpha(x)}\right) \mathrm{d} x \\
& -\int_{\partial \Omega} \frac{t^{q(x)+r(x)}}{q(x)+r(x)} c(x)|u|^{q(x)}|v|^{r(x)} \mathrm{d} x
\end{aligned}
$$

which gives

$$
\begin{aligned}
\Phi_{u, v}^{\prime}(t)= & \int_{\Omega} t^{p(x)-1}\left(|\nabla u|^{p(x)}+|u|^{p(x)}\right) \mathrm{d} x+\int_{\Omega} t^{p(x)-1}\left(|\nabla v|^{p(x)}+|v|^{p(x)}\right) \mathrm{d} x \\
& -\int_{\Omega} t^{-\alpha(x)}\left(\lambda a(x)|u|^{1-\alpha(x)}+\mu b(x)|v|^{1-\alpha(x)}\right) \mathrm{d} x \\
& -\int_{\partial \Omega} t^{q(x)+r(x)-1} c(x)|u|^{q(x)}|v|^{r(x)} \mathrm{d} x
\end{aligned}
$$

and

$$
\begin{aligned}
\Phi_{u, v}^{\prime \prime}(t)= & \int_{\Omega}(p(x)-1) t^{p(x)-2}\left(|\nabla u|^{p(x)}+|u|^{p(x)}\right) \mathrm{d} x \\
& +\int_{\Omega}(p(x)-1) t^{p(x)-2}\left(|\nabla v|^{p(x)}+|v|^{p(x)}\right) \mathrm{d} x \\
& +\int_{\Omega} \alpha(x) t^{-\alpha(x)-1}\left(\lambda a(x)|u|^{1-\alpha(x)}+\mu b(x)|v|^{1-\alpha(x)}\right) \mathrm{d} x \\
& -\int_{\partial \Omega}(q(x)+r(x)-1) t^{q(x)+r(x)-2} c(x)|u|^{q(x)}|v|^{r(x)} \mathrm{d} x .
\end{aligned}
$$

Such maps are called fiber maps and were introduced by Drabek and Pohozaev in 6 .

By (2.1), 2.2), 2.3), and Theorems 2.2, 2.3, 2.4 and 2.5, we obtain that

$$
\begin{aligned}
P(u, v) & =\int_{\Omega}\left(\lambda a(x)\left(u^{+}\right)^{1-\alpha(x)}+\mu b(x)\left(v^{+}\right)^{1-\alpha(x)}\right) \mathrm{d} x \\
& \leq 2 \lambda|a(x)|_{1-\alpha(x)}\left|\left(u^{+}\right)^{1-\alpha(x)}\right|_{\frac{p^{*}(x)}{1-\alpha(x)}}+2 \mu|b(x)|_{1-\alpha(x)}\left|\left(v^{+}\right)^{1-\alpha(x)}\right|_{\frac{p^{*}(x)}{1-\alpha(x)}} \\
& \leq 2 \lambda|a(x)|_{r_{1}(x)}\left(\left|\left(u^{+}\right)\right|_{p^{*}(x)}\right)^{1-\alpha_{0}}+2 \mu|b(x)|_{r_{2}(x)}\left(\left|\left(v^{+}\right)\right|_{p^{*}(x)}\right)^{1-\alpha_{0}} \\
& \leq 2 \lambda c_{1}\left\|u^{+}\right\|_{p(x)}^{1-\alpha_{0}}+2 \mu c_{2}\left\|v^{+}\right\|_{p(x)}^{1-\alpha_{0}} \\
& \leq \lambda c_{3}\left\|(u, v)^{+}\right\|^{1-\alpha^{-}}+\mu c_{4}\left\|(u, v)^{+}\right\|^{1-\alpha^{-}} \\
& \leq c_{5}(\lambda+\mu)\left\|(u, v)^{+}\right\|^{1-\alpha^{-}}
\end{aligned}
$$


and

$$
\begin{aligned}
Q(u, v)=\int_{\partial \Omega} c(x)|u|^{q(x)}|v|^{r(x)} \mathrm{d} x & \leq\left.\left.\left.\left. 3|c(x)|_{r_{3}(x)}|| u\right|^{q(x)}\right|_{\frac{p^{*}(x)}{q(x)}}|| v\right|^{r(x)}\right|_{\frac{p^{*}(x)}{r(x)}} \\
& \leq 3|c(x)|_{r_{3}(x)}\left(|u|_{p^{*}(x)}\right)^{q_{0}}\left(|v|_{p^{*}(x)}\right)^{r_{0}} \\
& \leq c_{6}\|u\|_{p(x)}^{q_{0}}\|v\|_{p(x)}^{r_{0}} \\
& \leq c_{7}\|(u, v)\|^{q^{+}+r^{+}} .
\end{aligned}
$$

Lemma 3.2. Let $(u, v) \in W \backslash\{(0,0)\}$. Then $(t u, t v) \in \mathcal{N}_{\lambda, \mu}$ if and only if $\Phi_{u, v}^{\prime}(t)=$ 0 for all $t>0$.

Proof. The result is a consequence of the fact that

$$
\Phi_{u, v}^{\prime}(t)=\left\langle I_{\lambda, \mu}^{\prime}(t u, t v),(u, v)\right\rangle .
$$

From Lemma 3.2 we have that the elements in $\mathcal{N}_{\lambda, \mu}$ correspond to stationary points of the maps $\Phi_{u, v}(t)$ and, in particular, $(u, v) \in \mathcal{N}_{\lambda, \mu}$ if and only if $\Phi_{u, v}^{\prime}(1)=$ 0 . Hence, it is natural to split $\mathcal{N}_{\lambda, \mu}$ into three parts corresponding to local minima, local maxima, and points of inflection $\Phi_{u, v}(t)$ defined as follows:

$$
\begin{aligned}
\mathcal{N}_{\lambda, \mu}^{+} & =\left\{(u, v) \in \mathcal{N}_{\lambda, \mu}: \Phi_{u, v}^{\prime \prime}(1)>0\right\} \\
& =\left\{(t u, t v) \in W \backslash\{0,0\}: \Phi_{u, v}^{\prime}(t)=0, \Phi_{u, v}^{\prime \prime}(t)>0\right\}, \\
\mathcal{N}_{\lambda, \mu}^{-} & =\left\{(u, v) \in \mathcal{N}_{\lambda, \mu}: \Phi_{u, v}^{\prime \prime}(1)<0\right\} \\
& =\left\{(t u, t v) \in W \backslash\{0,0\}: \Phi_{u, v}^{\prime}(t)=0, \Phi_{u, v}^{\prime \prime}(t)<0\right\}, \\
\mathcal{N}_{\lambda, \mu}^{0} & =\left\{(u, v) \in \mathcal{N}_{\lambda, \mu}: \Phi_{u, v}^{\prime \prime}(1)=0\right\} \\
& =\left\{(t u, t v) \in W \backslash\{0,0\}: \Phi_{u, v}^{\prime}(t)=0, \Phi_{u, v}^{\prime \prime}(t)=0\right\} .
\end{aligned}
$$

Our first result is the following.

Lemma 3.3. $E_{\lambda, \mu}$ is coercive and bounded below on $\mathcal{N}_{\lambda, \mu}$.

Proof. Suppose that $(u, v) \in \mathcal{N}_{\lambda, \mu}$ and $\|(u, v)\|>1$. Without loss of generality, we may assume that $\|u\|_{p(x)},\|u\|_{q(x)}>1$. Therefore, using 2.2 -2.4) and (3.1) we estimate $E_{\lambda, \mu}(u, v)$ as follows:

$$
\begin{aligned}
& E_{\lambda, \mu}(u, v) \\
&=\int_{\Omega}\left(\frac{|\nabla u|^{p(x)}}{p(x)}+\frac{|u|^{p(x)}}{p(x)}\right) \mathrm{d} x+\int_{\Omega}\left(\frac{|\nabla v|^{p(x)}}{p(x)}+\frac{|v|^{p(x)}}{p(x)}\right) \mathrm{d} x \\
&-\int_{\Omega}\left(\frac{\lambda a(x)}{1-\alpha(x)}|u|^{1-\alpha(x)}+\frac{\mu b(x)}{1-\alpha(x)}|v|^{1-\alpha(x)}\right) \mathrm{d} x \\
&-\int_{\partial \Omega} \frac{c(x)}{q(x)+r(x)}|u|^{q(x)}|v|^{r(x)} \mathrm{d} x \\
& \geq \frac{1}{p^{+}}\left(\int_{\Omega}|\nabla u|^{p(x)}+|u|^{p(x)}\right) \mathrm{d} x+\frac{1}{p^{+}}\left(\int_{\Omega}|\nabla v|^{p(x)}+|v|^{p(x)}\right) \mathrm{d} x \\
&-\frac{1}{1-\alpha^{+}} \int_{\Omega}\left(\lambda a(x)|u|^{1-\alpha(x)}+\mu b(x)|v|^{1-\alpha(x)}\right) \mathrm{d} x
\end{aligned}
$$




$$
\begin{aligned}
& -\frac{1}{q^{-}+r^{-}} \int_{\partial \Omega} c(x)|u|^{q(x)}|v|^{r(x)} \mathrm{d} x \\
\geq & \left(\frac{1}{p^{+}}-\frac{1}{q^{-}+r^{-}}\right)\left(\int_{\Omega}\left(|\nabla u|^{p(x)}+|u|^{p(x)}\right) \mathrm{d} x+\int_{\Omega}\left(|\nabla v|^{p(x)}+|v|^{p(x)}\right) \mathrm{d} x\right) \\
& -\left(\frac{1}{1-\alpha^{+}}-\frac{1}{q^{-}+r-}\right) \int_{\Omega}\left(\lambda a(x)|u|^{1-\alpha(x)}+\mu b(x)|v|^{1-\alpha(x)}\right) \mathrm{d} x \\
\geq & \left(\frac{1}{p^{+}}-\frac{1}{q^{-}+r^{-}}\right)\|(u, v)\|^{p^{-}}-c_{7}\left(\frac{1}{1-\alpha^{+}}-\frac{1}{q^{-}+r^{-}}\right)\|(u, v)\|^{1-\alpha^{-}} .
\end{aligned}
$$

Hence, since $p^{-}>1-\alpha^{-}$, we have $E_{\lambda, \mu}(u, v) \rightarrow \infty$ as $\|(u, v)\| \rightarrow \infty$. This implies that $E_{\lambda, \mu}$ is coercive and bounded below.

Lemma 3.4. Let $(u, v)$ be a local minimizer for $E_{\lambda, \mu}$ on subsets $\mathcal{N}_{\lambda, \mu}^{+}$or $\mathcal{N}_{\lambda, \mu}^{-}$of $\mathcal{N}_{\lambda, \mu}$ such that $(u, v) \notin \mathcal{N}_{\lambda, \mu}^{0}$. Then $(u, v)$ is a critical point of $E_{\lambda, \mu}$.

Proof. Since $(u, v)$ is a local minimizer for $E_{\lambda, \mu}$ under the constraint

$$
I_{\lambda, \mu}(u):=\left\langle E_{\lambda, \mu}^{\prime}(u, v),(u, v)\right\rangle=0,
$$

applying the theory of Lagrange multipliers we get the existence of $\sigma \in \mathbb{R}$ such that

$$
E_{\lambda, \mu}^{\prime}(u, v)=\sigma I_{\lambda, \mu}^{\prime}(u, v)
$$

So we have

$$
\left\langle E_{\lambda, \mu}^{\prime}(u, v),(u, v)\right\rangle=\sigma\left\langle I_{\lambda, \mu}^{\prime}(u, v),(u, v)\right\rangle=\sigma \Phi_{u, v}^{\prime \prime}(1)=0 .
$$

Yet $(u, v) \notin \mathcal{N}_{\lambda, \mu}^{0}$ and so $\Phi_{u, v}^{\prime \prime}(1) \neq 0$. Hence $\sigma=0$, and this completes the proof.

Now, we prove the following crucial lemma.

Lemma 3.5. There exists

$$
\Lambda_{0}=\frac{c_{8}}{c_{9}}\left(\frac{p^{+}+\alpha^{+}-1}{q^{-}+r^{+}+\alpha^{+}-1}\right)^{\frac{p^{+}+\alpha^{+}-1}{q^{-+r^{+}-p^{+}}}}\left(\frac{p^{+}-q^{-}-r^{+}}{q^{-}+r^{+}+\alpha^{+}-1}\right)
$$

such that for $0<\lambda+\mu<\Lambda_{0}$ we have $\mathcal{N}_{\lambda, \mu}^{ \pm} \neq \emptyset$ and $\mathcal{N}_{\lambda, \mu}^{0}=\{0\}$.

Proof. Firstly, using Lemma 3.4 we conclude that $\mathcal{N}_{\lambda, \mu}^{ \pm}$are non-empty for all $(\lambda, \mu)$ with $0<\lambda+\mu<\Lambda_{0}$. Now, we proceed by contradiction to prove that $\mathcal{N}_{\lambda, \mu}^{0}=\{0\}$ for all $(\lambda, \mu)$ with $0<\lambda+\mu<\Lambda_{0}$. Let us suppose that there exists $(u, v) \in \mathcal{N}_{\lambda, \mu}^{0}$ such that $\|(u, v)\|>1$. Then, from the definition of $\mathcal{N}_{\lambda, \mu}^{0}$, it follows that

$$
\begin{array}{r}
\int_{\Omega}\left(|\nabla u|^{p(x)}+|u|^{p(x)}\right) \mathrm{d} x+\int_{\Omega}\left(|\nabla v|^{p(x)}+|v|^{p(x)}\right) \mathrm{d} x-\lambda \int_{\Omega} a(x)|u|^{1-\alpha(x)} \mathrm{d} x \\
-\mu \int_{\Omega} b(x)|v|^{1-\alpha(x)} \mathrm{d} x-\int_{\partial \Omega} c(x)|u|^{q(x)}|v|^{r(x)} \mathrm{d} x=0
\end{array}
$$


So, using 3.4 combined with 3.3, we obtain

$$
\begin{aligned}
0= & \left\langle I_{\lambda, \mu}^{\prime}(u, v),(u, v)\right\rangle \\
= & \int_{\Omega} p(x)\left(|\nabla u|^{p(x)}+|u|^{p(x)}\right) \mathrm{d} x+\int_{\Omega} p(x)\left(|\nabla v|^{p(x)}+|v|^{p(x)}\right) \mathrm{d} x \\
& -\lambda \int_{\Omega} a(x)(1-\alpha(x))|u|^{1-\alpha(x)} \mathrm{d} x-\mu \int_{\Omega} b(x)(1-\alpha(x))|v|^{1-\alpha(x)} \mathrm{d} x \\
& -\int_{\partial \Omega} c(x)(q(x)+r(x))|u|^{q(x)}|v|^{r(x)} \mathrm{d} x \\
\geq & p^{-} \int_{\Omega}\left(|\nabla u|^{p(x)}+|u|^{p(x)}\right) \mathrm{d} x+p^{-} \int_{\Omega}\left(|\nabla v|^{p(x)}+|v|^{p(x)}\right) \mathrm{d} x \\
& -\left(1-\alpha^{-}\right) \int_{\Omega}\left(\lambda a(x)|u|^{1-\alpha(x)}+\mu b(x)|v|^{1-\alpha(x)}\right) \mathrm{d} x \\
& -\left(q^{+}+r^{+}\right) \int_{\partial \Omega} c(x)|u|^{q(x)}|v|^{r(x)} \mathrm{d} x \\
\geq & \left(p^{-}-\left(1-\alpha^{-}\right)\right)\left(\int_{\Omega}\left(|\nabla u|^{p(x)}+|u|^{p(x)}\right) \mathrm{d} x+\int_{\partial \Omega}\left(|\nabla v|^{p(x)}+|v|^{p(x)}\right) \mathrm{d} x\right) \\
& +\left(1-\alpha^{-}-q^{+}-r^{+}\right) \int_{\partial \Omega} c(x)|u|^{q(x)}|v|^{r(x)} \mathrm{d} x .
\end{aligned}
$$

Using 3.2 we obtain that

$$
\left(p^{-}+\alpha^{-}-1\right)\|(u, v)\|^{p^{-}}+c_{8}\left(1-\alpha^{-}-q^{+}-r^{+}\right)\|(u, v)\|^{q^{+}+r^{+}} \leq 0,
$$

which implies that

$$
\|(u, v)\| \geq \frac{1}{c_{8}}\left(\frac{p^{-}+\alpha^{-}-1}{q^{+}+r^{+}+\alpha^{+}-1}\right)^{\frac{1}{q^{+}+r^{+}-p^{-}}} .
$$

Similarly, since $(u, v) \in \mathcal{N}_{\lambda, \mu}$, we have

$$
\begin{aligned}
\int_{\Omega}\left(|\nabla u|^{p(x)}+|u|^{p(x)}\right) & \mathrm{d} x+\int_{\Omega}\left(|\nabla v|^{p(x)}+|v|^{p(x)}\right) \mathrm{d} x-\lambda \int_{\Omega} a(x)|u|^{1-\alpha(x)} \mathrm{d} x \\
& -\mu \int_{\Omega} b(x)|v|^{1-\alpha(x)} \mathrm{d} x-\int_{\partial \Omega} c(x)|u|^{q(x)}|v|^{r(x)} \mathrm{d} x=0,
\end{aligned}
$$

and since $(u, v) \in \mathcal{N}_{\lambda, \mu}^{0}$, we get

$$
\begin{aligned}
p^{+} \int_{\Omega} & \left(|\nabla u|^{p(x)}+|u|^{p(x)}\right) \mathrm{d} x+p^{+} \int_{\Omega}\left(|\nabla v|^{p(x)}+|v|^{p(x)}\right) \mathrm{d} x \\
& -\left(1-\alpha^{+}\right) \int_{\Omega}\left(\lambda a(x)|u|^{1-\alpha(x)}+\mu b(x)|v|^{1-\alpha(x)}\right) \mathrm{d} x \\
& -\left(q^{-}+r^{+}\right) \int_{\partial \Omega} c(x)|u|^{q(x)}|v|^{r(x)} \mathrm{d} x \geq 0 .
\end{aligned}
$$


Therefore,

$$
\begin{aligned}
& \left(p^{+}-q^{-}-r^{+}\right)\left(\int_{\Omega}\left(|\nabla u|^{p(x)}+|u|^{p(x)}\right) \mathrm{d} x+\int_{\Omega}\left(|\nabla v|^{p(x)}+|v|^{p(x)}\right) \mathrm{d} x\right) \\
& \quad+\left(q^{-}+r^{+}+\alpha^{+}-1\right)\left(\int_{\Omega} \lambda a(x)|u|^{1-\alpha(x)} \mathrm{d} x+\int_{\Omega} \mu b(x)|v|^{1-\alpha(x)} \mathrm{d} x\right) \geq 0 .
\end{aligned}
$$

Now using 3.1 we get

$$
\left(p^{+}-q^{-}-r^{+}\right)\|(u, v)\|^{p^{-}}+c_{9}(\lambda+\mu)\left(q^{-}+r^{+}+\alpha^{+}-1\right)\|(u, v)\|^{1-\alpha^{+}} \geq 0,
$$

and hence

$$
\|(u, v)\| \leq c_{9}\left((\lambda+\mu) \frac{q^{-}+r^{+}+\alpha^{+}-1}{p^{+}-q^{-}-r^{+}}\right)^{\frac{1}{p^{++\alpha+}-1}} .
$$

From 3.5 and 3.6 ,

$$
c_{9}(\lambda+\mu)\left(\frac{q^{-}+r^{+}+\alpha^{+}-1}{p^{+}-q^{-}-r^{+}}\right) \geq c_{8}\left(\frac{p^{+}+\alpha^{+}-1}{q^{-}+r^{+}+\alpha^{+}-1}\right)^{\frac{p^{+}+\alpha^{+}-1}{q^{-}+r^{+}-p^{+}}}
$$

and so

$$
\lambda+\mu \geq \frac{c_{8}}{c_{9}}\left(\frac{p^{+}+\alpha^{+}-1}{q^{-}+r^{+}+\alpha^{+}-1}\right)^{\frac{p^{+}+\alpha^{+}-1}{q^{-+r^{+}-p^{+}}}}\left(\frac{p^{+}-q^{-}-r^{+}}{q^{-}+r^{+}+\alpha^{+}-1}\right)=\Lambda_{0} .
$$

Therefore $\lambda+\mu \geq \Lambda_{0}$, which is impossible. Thus, $\mathcal{N}_{\lambda, \mu}^{0}=\{0\}$ for all $\lambda, \mu$ with $0<\lambda+\mu<\Lambda_{0}$, and the proof is complete.

By Lemmas 3.3 and 3.4 for $0<\lambda+\mu<\Lambda_{0}$ we can write $\mathcal{N}_{\lambda, \mu}=\mathcal{N}_{\lambda, \mu}^{+} \cup \mathcal{N}_{\lambda, \mu}^{-}$ and define

$$
c_{\lambda, \mu}^{+}=\inf _{(u, v) \in \mathcal{N}_{\lambda, \mu}^{+}} E_{\lambda, \mu}(u, v) \quad \text { and } \quad c_{\lambda, \mu}^{-}=\inf _{(u, v) \in \mathcal{N}_{\lambda, \mu}^{-}} E_{\lambda, \mu}(u, v) .
$$

\section{Existence of Minimizer on $\mathcal{N}_{\lambda, \mu}^{+}$}

In this section, we will show that the minimum of $E_{\lambda, \mu}$ is achieved in $\mathcal{N}_{\lambda, \mu}^{+}$. Also, we show that this minimizer is also the first solution of problem (1.1).

Lemma 4.1. If $0<\lambda+\mu<\Lambda_{0}$, then $c_{\lambda, \mu}^{+}<0$ for all $(u, v) \in \mathcal{N}_{\lambda, \mu}^{+}$.

Proof. Let $\left(u_{0}^{+}, v_{0}^{+}\right) \in \mathcal{N}_{\lambda, \mu}^{+}$. Then we have $\phi_{u_{0}^{+}, v_{0}^{+}}^{\prime \prime}(1)>0$, which gives

$$
\begin{aligned}
p^{+} \int_{\Omega} & \left(|\nabla u|^{p(x)}+|u|^{p(x)}\right) \mathrm{d} x+p^{+}\left(\int_{\Omega}|\nabla v|^{p(x)}+|v|^{p(x)}\right) \mathrm{d} x \\
& -\left(1-\alpha^{+}\right)\left(\lambda \int_{\Omega} a(x)|u|^{1-\alpha(x)} \mathrm{d} x+\mu \int_{\Omega} b(x)|v|^{1-\alpha(x)} \mathrm{d} x\right) \\
& -\left(q^{+}+r^{+}\right) \int_{\partial \Omega} c(x)|u|^{q(x)}|v|^{r(x)} \mathrm{d} x>0 .
\end{aligned}
$$


On the other hand, from the definition of $E_{\lambda, \mu}$, we can write

$$
\begin{aligned}
E_{\lambda, \mu}(u, v) \leq & \frac{1}{p^{-}}\left(\int_{\Omega}\left(|\nabla u|^{p(x)}+|u|^{p(x)}\right) \mathrm{d} x+\left(\int_{\Omega}|\nabla v|^{p(x)}+|v|^{p(x)}\right) \mathrm{d} x\right) \\
& -\frac{1}{1-\alpha^{+}}\left(\lambda \int_{\Omega} a(x)|u|^{1-\alpha(x)} \mathrm{d} x+\mu \int_{\Omega} b(x)|v|^{1-\alpha(x)} \mathrm{d} x\right) \\
& -\frac{1}{q^{+}+r^{+}} \int_{\partial \Omega} c(x)|u|^{q(x)}|v|^{r(x)} \mathrm{d} x .
\end{aligned}
$$

Now, we multiply 3.3 by $-\left(1-\alpha^{+}\right)$and obtain

$$
\begin{aligned}
-\left(1-\alpha^{+}\right)( & \left.\int_{\Omega}\left(|\nabla u|^{p(x)}+|u|^{p(x)}\right) \mathrm{d} x+\left(\int_{\Omega}|\nabla v|^{p(x)}+|v|^{p(x)}\right) \mathrm{d} x\right) \\
& +\lambda\left(1-\alpha^{+}\right) \int_{\Omega} a(x)|u|^{1-\alpha(x)} \mathrm{d} x+\mu\left(1-\alpha^{+}\right) \int_{\Omega} b(x)|v|^{1-\alpha(x)} \mathrm{d} x \\
& +\left(1-\alpha^{+}\right) \int_{\partial \Omega} c(x)|u|^{q(x)}|v|^{r(x)} \mathrm{d} x=0 .
\end{aligned}
$$

Adding the above equality with (4.1), we get

$$
\begin{aligned}
& \int_{\partial \Omega} c(x)|u|^{q(x)}|v|^{r(x)} \mathrm{d} x \\
& \quad<\frac{p^{+}+\alpha^{+}-1}{q^{+}+r^{+}+\alpha^{+}-1}\left(\int_{\Omega}\left(|\nabla u|^{p(x)}+|u|^{p(x)}\right) \mathrm{d} x+\left(\int_{\Omega}|\nabla v|^{p(x)}+|v|^{p(x)}\right) \mathrm{d} x\right) .
\end{aligned}
$$

Moreover, using (3.3) with 4.2), we have

$$
\begin{aligned}
& E_{\lambda, \mu}(u, v) \\
& \leq \quad\left(\frac{1}{p^{-}}-\frac{1}{1-\alpha^{+}}\right)\left(\int_{\Omega}\left(|\nabla u|^{p(x)}+|u|^{p(x)}\right) \mathrm{d} x+\left(\int_{\Omega}|\nabla v|^{p(x)}+|v|^{p(x)}\right) \mathrm{d} x\right) \\
& \quad-\left(\frac{1}{q^{+}+r^{+}}-\frac{1}{1-\alpha^{+}}\right) \int_{\partial \Omega} c(x)|u|^{q(x)}|v|^{r(x)} \mathrm{d} x .
\end{aligned}
$$

Hence, using 4.3 and 4.4, we obtain

$$
E_{\lambda, \mu}(u, v)<-\frac{\left(p^{-}+\alpha^{+}-1\right)\left(q^{+}+r^{+}-p^{-}\right)}{p^{-}\left(1-\alpha^{+}\right)\left(q^{+}+r^{+}\right)}\|(u, v)\|^{p^{-}}<0
$$

Therefore, $c_{\lambda, \mu}^{+}<0$ follows from the definition of $c_{\lambda, \mu}^{+}$, and the proof is complete. 
Theorem 4.2. If $\lambda, \mu$ are such that $0<\lambda+\mu<\Lambda_{0}$, then the functional $I_{\lambda, \mu}$ has a minimizer $\left(u_{0}^{-}, v_{0}^{-}\right) \in \mathcal{N}_{\lambda, \mu}^{+}$satisfying

$$
E_{\lambda, \mu}\left(u_{0}^{+}, v_{0}^{+}\right)=\inf _{(u, v) \in \mathcal{N}_{\lambda, \mu}^{+}} E_{\lambda, \mu}(u, v)
$$

Proof. Since $E_{\lambda, \mu}$ is bounded below on $\mathcal{N}_{\lambda, \mu}$, and so on $\mathcal{N}_{\lambda, \mu}^{+}$, there exists a sequence $\left\{\left(u_{n}^{+}, v_{n}^{+}\right)\right\} \subset \mathcal{N}_{\lambda, \mu}^{+}$such that $E_{\lambda, \mu}\left(u_{n}^{+}, v_{n}^{+}\right) \rightarrow \inf _{(u, v) \in \mathcal{N}_{\lambda, \mu}^{+}} E_{\lambda, \mu}(u, v)$ as $n \rightarrow \infty$. Since $E_{\lambda, \mu}$ is coercive, $\left\{\left(u_{n}, v_{n}\right)\right\}$ is bounded in $W$. Thus, we may assume that, without loss of generality, $\left(u_{n}^{+}, v_{n}^{+}\right) \rightarrow\left(u_{0}^{+}, v_{0}^{+}\right)$weakly in $W$, and by the compact embedding we have

$$
\begin{aligned}
& u_{n}^{+} \rightarrow u_{0}^{+} \quad \text { in } L_{a(x)}^{1-\alpha(x)}(\Omega) \text { and in } L_{b(x)}^{q(x)+r(x)}(\partial \Omega), \\
& v_{n}^{+} \rightarrow v_{0}^{+} \quad \text { in } L_{a(x)}^{1-\alpha(x)}(\Omega) \text { and in } L_{b(x)}^{q(x)+r(x)}(\partial \Omega) .
\end{aligned}
$$

This implies that

$$
\begin{aligned}
& P\left(u_{n}^{+}, v_{n}^{+}\right) \rightarrow P\left(u_{0}^{+}, v_{0}^{+}\right) \quad \text { as } n \rightarrow \infty \\
& Q\left(u_{n}^{+}, v_{n}^{+}\right) \rightarrow Q\left(u_{0}^{+}, v_{0}^{+}\right) \quad \text { as } n \rightarrow \infty
\end{aligned}
$$

Now, we shall prove that $u_{n}^{+} \rightarrow u_{0}^{+}$strongly in $W^{1, p(x)}(\Omega)$ and $v_{n}^{+} \rightarrow v_{0}^{+}$strongly in $W^{1, p(x)}(\Omega)$. Suppose otherwise; then either

$$
\left\|u_{0}^{+}\right\|_{p} \leq \liminf _{n \rightarrow \infty} \int_{\Omega}\left\|u_{n}^{+}\right\|_{p} \quad \text { or } \quad\left\|v_{0}^{+}\right\|_{p} \leq \liminf _{n \rightarrow \infty} \int_{\Omega}\left\|v_{n}^{+}\right\|_{p} .
$$

By the compact embeddings and using (3.3), we can write

$$
\begin{aligned}
E_{\lambda, \mu}\left(u_{n}^{+}, v_{n}^{+}\right) \geq & \left(\frac{1}{p^{-}}-\frac{1}{q^{+}+r^{+}}\right) \\
& \times\left(\int_{\Omega}\left(\left|\nabla u_{n}^{+}\right|^{p(x)}+\left|u_{n}^{+}\right|^{p(x)}\right) \mathrm{d} x\left(\int_{\Omega}\left|\nabla v_{n}^{+}\right|^{p(x)}+\left|v_{n}^{+}\right|^{p(x)}\right) \mathrm{d} x\right) \\
+ & \left(\frac{1}{q^{+}+r^{+}}-\frac{1}{1-\alpha^{+}}\right) \\
& \times\left(\lambda \int_{\Omega} a(x)\left|u_{n}^{+}\right|^{1-\alpha(x)} \mathrm{d} x+\mu \int_{\Omega} b(x)\left|v_{n}^{+}\right|^{1-\alpha(x)} \mathrm{d} x\right) .
\end{aligned}
$$


Letting $n$ tend to $\infty$, we get

$$
\begin{aligned}
\lim _{n \rightarrow \infty} E_{\lambda, \mu}\left(u_{n}^{+}, v_{n}^{+}\right) \\
\geq\left(\frac{1}{p^{-}}-\frac{1}{q^{+}+r^{+}}\right) \\
\quad \times \lim _{n \rightarrow \infty}\left(\int_{\Omega}\left(\left|\nabla u_{n}^{+}\right|^{p(x)}+\left|u_{n}^{+}\right|^{p(x)}\right) \mathrm{d} x+\left(\int_{\Omega}\left|\nabla v_{n}^{+}\right|^{p(x)}+\left|v_{n}^{+}\right|^{p(x)}\right) \mathrm{d} x\right) \\
+\left(\frac{1}{q^{+}+r^{+}}-\frac{1}{1-\alpha^{+}}\right) \\
\quad \times \lim _{n \rightarrow \infty}\left(\lambda \int_{\Omega} a(x)\left|u_{n}^{+}\right|^{1-\alpha(x)} \mathrm{d} x+\mu \int_{\Omega} b(x)\left|v_{n}^{+}\right|^{1-\alpha(x)} \mathrm{d} x\right) .
\end{aligned}
$$

Therefore, using (3.1) we obtain

$$
\begin{aligned}
c_{\lambda, \mu}^{+} & =\inf _{(u, v) \in \mathcal{N}^{+}} E_{\lambda, \mu}(u, v) \\
& >\left(\frac{1}{p^{-}}-\frac{1}{q^{+}+r^{+}}\right)\left\|\left(u_{0}^{+}, v_{0}^{+}\right)\right\|^{p^{-}}-c_{7}\left(\frac{1}{q^{+}+r^{+}}-\frac{1}{1-\alpha^{+}}\right)\left\|\left(u_{0}^{+}, v_{0}^{+}\right)\right\|^{1-\alpha^{+}} \\
& >0,
\end{aligned}
$$

since $p^{-}>1-\alpha^{+}$and $\left\|\left(u_{0}^{+}, v_{0}^{+}\right)\right\|>1$, which gives a contradiction. Thus, $u_{n}^{+} \rightarrow u_{0}^{+}$ strongly in $W^{1, p(x)}(\Omega), v_{n}^{+} \rightarrow v_{0}^{+}$strongly in $W^{1, p(x)}(\Omega), u_{n} \rightarrow u_{0}$ strongly in $W^{1, p(x)}(\Omega)$, and $E_{\lambda, \mu}\left(u_{0}^{+}, v_{0}^{+}\right)=\inf _{(u, v) \in \mathcal{N}_{\lambda, \mu}^{+}} E_{\lambda, \mu}(u, v)$, which completes the proof of the theorem.

\section{EXISTENCE OF Minimizer ON $\mathcal{N}_{\lambda, \mu}^{-}$}

In this section, we shall show the existence of a second solution of problem 1.1 by proving the existence of a minimizer of $E_{\lambda, \mu}$ on $\mathcal{N}_{\lambda, \mu}^{-}$.

Lemma 5.1. If $0<\lambda+\mu<\Lambda_{0}$, then $c_{\lambda, \mu}^{-}>0$ for all $(u, v) \in \mathcal{N}_{\lambda, \mu}^{-}$.

Proof. Let $\left(u_{0}^{-}, v_{0}^{-}\right) \in \mathcal{N}_{\lambda, \mu}^{-}$. Then we have, from 3.3 ,

$$
\begin{gathered}
\int_{\Omega}\left(|\nabla u|^{p(x)}+|u|^{p(x)}\right) \mathrm{d} x+\left(\int_{\Omega}|\nabla v|^{p(x)}+|v|^{p(x)}\right) \mathrm{d} x \\
-\lambda \int_{\Omega} a(x)|u|^{1-\alpha(x)} \mathrm{d} x+\mu \int_{\Omega} b(x)|v|^{1-\alpha(x)} \mathrm{d} x \\
-\int_{\partial \Omega} c(x)|u|^{q(x)}|v|^{r(x)} \mathrm{d} x=0 .
\end{gathered}
$$


On the other hand, from the definition of the $E_{\lambda}$, we can write

$$
\begin{aligned}
E_{\lambda, \mu}(u, v) \geq & \frac{1}{p^{-}}\left(\int_{\Omega}\left(|\nabla u|^{p(x)}+|u|^{p(x)}\right) \mathrm{d} x+\left(\int_{\Omega}|\nabla v|^{p(x)}+|v|^{p(x)}\right) \mathrm{d} x\right) \\
& -\frac{\lambda}{1-\alpha^{+}} \int_{\Omega} a(x)|u|^{1-\alpha(x)} \mathrm{d} x-\frac{\mu}{1-\alpha^{+}} \int_{\Omega} b(x)|v|^{1-\alpha(x)} \mathrm{d} x \\
& -\frac{1}{q^{+}+r^{+}} \int_{\partial \Omega} c(x)|u|^{q(x)}|v|^{r(x)} \mathrm{d} x .
\end{aligned}
$$

Therefore, using (5.1), (5.2), and (3.1) we get

$$
\begin{aligned}
& E_{\lambda, \mu}(u, v) \\
& \geq\left(\frac{1}{p^{-}}-\frac{1}{q^{+}+r^{+}}\right)\left(\int_{\Omega}\left(|\nabla u|^{p(x)}+|u|^{p(x)}\right) \mathrm{d} x+\left(\int_{\Omega}|\nabla v|^{p(x)}+|v|^{p(x)}\right) \mathrm{d} x\right) \\
&+\left(\frac{1}{q^{+}+r^{+}}-\frac{1}{1-\alpha^{+}}\right)\left(\int_{\Omega} \lambda a(x)|u|^{1-\alpha(x)} \mathrm{d} x+\int_{\Omega} \mu b(x)|v|^{1-\alpha(x)} \mathrm{d} x\right) \\
& \geq\left(\frac{1}{p^{-}}-\frac{1}{q^{+}+r^{+}}\right)\|(u, v)\|^{p^{-}}+c_{5}\left(\frac{1}{q^{+}+r^{+}}-\frac{1}{1-\alpha^{+}}\right)(\lambda+\mu)\|(u, v)\|^{1-\alpha^{+}} \\
& \geq {\left[\left(\frac{1}{p^{-}}-\frac{1}{q^{+}}\right)+c_{7}\left(\frac{1}{q^{+}}-\frac{1}{2-\alpha^{+}-\beta^{+}}\right)\right]\|(u, v)\|^{p^{-}}>0, }
\end{aligned}
$$

since $p^{-}>1-\alpha^{+}$. Consequently, $\inf _{(u, v) \in \mathcal{N}_{\lambda, \mu}^{-}} E_{\lambda, \mu}(u, v)>0$. Indeed, if this infimum is zero, then from 5.3 , the minimizing sequence $\left\{\left(u_{k}, v_{k}\right)\right\}$ converges strongly in $W$ to $(0,0)$ and $(0,0) \notin \mathcal{N}_{\lambda, \mu}^{-}$, a contradiction. Therefore $c_{\lambda, \mu}^{-}>0$ follows from the definition of $c_{\lambda, \mu}^{-}$, and the proof is complete.

Theorem 5.2. If $\lambda, \mu$ are such that $0<\lambda+\mu<\Lambda_{0}$, then the functional $I_{\lambda, \mu}$ has a minimizer $\left(u_{0}^{-}, v_{0}^{-}\right) \in \mathcal{N}_{\lambda, \mu}^{-}$satisfying $E_{\lambda, \mu}\left(u_{0}^{-}, v_{0}^{-}\right)=\inf _{(u, v) \in \mathcal{N}_{\lambda, \mu}^{-}} E_{\lambda, \mu}(u, v)$.

Proof. Since $E_{\lambda, \mu}$ is bounded below on $\mathcal{N}_{\lambda, \mu}$, and so on $\mathcal{N}_{\lambda, \mu}^{-}$, there exists a sequence $\left\{\left(u_{n}^{-}, v_{n}^{-}\right)\right\} \subset \mathcal{N}_{\lambda, \mu}^{-}$such that $E_{\lambda, \mu}\left(u_{n}^{-}, v_{n}^{-}\right) \rightarrow \inf _{(u, v) \in \mathcal{N}_{\lambda, \mu}^{-}} E_{\lambda, \mu}(u, v)$ as $n \rightarrow \infty$. Since $E_{\lambda, \mu}$ is coercive, $\left\{\left(u_{n}, v_{n}\right)\right\}$ is bounded in $W$. Thus, we may assume that, without loss of generality, $\left(u_{n}^{-}, v_{n}^{-}\right) \rightarrow\left(u_{0}^{-}, v_{0}^{-}\right)$weakly in $W$, and by the compact embedding we have

$$
\begin{aligned}
& u_{n}^{-} \rightarrow u_{0}^{-} \quad \text { in } L_{a(x)}^{1-\alpha(x)}(\Omega) \text { and in } L_{b(x)}^{q(x)+r(x)}(\Omega), \\
& v_{n}^{-} \rightarrow v_{0}^{-} \quad \text { in } L_{a(x)}^{1-\alpha(x)}(\Omega) \text { and in } L_{b(x)}^{q(x)+r(x)}(\Omega) .
\end{aligned}
$$

This implies that

$$
\begin{aligned}
& P\left(u_{n}^{-}, v_{n}^{-}\right) \rightarrow P\left(u_{0}^{-}, v_{0}^{-}\right) \quad \text { as } n \rightarrow \infty, \\
& Q\left(u_{n}^{-}, v_{n}^{-}\right) \rightarrow Q\left(u_{0}^{-}, v_{0}^{-}\right) \quad \text { as } n \rightarrow \infty
\end{aligned}
$$


Moreover, since $\mathcal{N}_{\lambda, \mu}^{+} \cap \mathcal{N}_{\lambda, \mu}^{-}=\emptyset$ and $\inf _{(u, v) \in \mathcal{N}_{\lambda, \mu}^{+}} E_{\lambda, \mu}(u, v)<0$, we obtain $(u, v) \in \mathcal{N}_{\lambda, \mu}^{-}$. On the other hand, if $\left(u_{0}^{-}, v_{0}^{-}\right) \in \mathcal{N}_{\lambda, \mu}^{-}$, there exists $t_{0}$ such that $\left(t_{0} u_{0}^{-}, t_{0} v_{0}^{-}\right) \in \mathcal{N}_{\lambda, \mu}^{-}$and so $E_{\lambda}\left(t_{0} u_{0}^{-}, t_{0} v_{0}^{-}\right) \leq E_{\lambda}\left(u_{0}^{-}, v_{0}^{-}\right)$. In fact, since

$$
\begin{aligned}
I_{\lambda, \mu}^{\prime}(u, v)= & \int_{\Omega} p(x)\left(|\nabla u|^{p(x)}+|u|^{p(x)}\right) \mathrm{d} x+\int_{\Omega} p(x)\left(|\nabla v|^{p(x)}+|v|^{p(x)}\right) \mathrm{d} x \\
& -\lambda \int_{\Omega} a(x)(1-\alpha(x))|u|^{1-\alpha(x)} \mathrm{d} x-\mu \int_{\Omega} b(x)(1-\alpha(x))|v|^{1-\alpha(x)} \mathrm{d} x \\
& -\int_{\partial \Omega} c(x)(q(x)+r(x))|u|^{q(x)}|v|^{r(x)} \mathrm{d} x,
\end{aligned}
$$

using $(3.2)$ we get

$$
\begin{aligned}
& I_{\lambda, \mu}^{\prime}\left(t_{0} u_{0}^{-}, t_{0} v_{0}^{-}\right)=\int_{\Omega} p(x)\left(\left|\nabla t_{0} u_{0}^{-}\right|^{p(x)}+\left|t_{0} u_{0}^{-}\right|^{p(x)}\right) \mathrm{d} x \\
& \quad+\int_{\Omega} p(x)\left(\left|\nabla t_{0} v_{0}^{-}\right|^{p(x)}+\left|t_{0} v_{0}^{-}\right|^{p(x)}\right) \mathrm{d} x \\
& \quad-\lambda \int_{\Omega} a(x)(1-\alpha(x))\left|t_{0} u_{0}^{-}\right|^{1-\alpha(x)} \mathrm{d} x-\mu \int_{\Omega} b(x)(1-\alpha(x))\left|t_{0} v_{0}^{-}\right|^{1-\alpha(x)} \mathrm{d} x \\
& \quad-\int_{\partial \Omega} c(x)(q(x)+r(x))\left|t_{0} u_{0}^{-}\right|^{q(x)}\left|t_{0} v_{0}^{-}\right|^{r(x)} \mathrm{d} x \\
& \leq t_{0}^{p^{+}} p^{+}\left(\int_{\Omega}\left(\left|\nabla u_{0}^{-}\right|^{p(x)}+\left|u_{0}^{-}\right|^{p(x)}\right) \mathrm{d} x+\int_{\Omega}\left(\left|\nabla v_{0}^{-}\right|^{p(x)}+\left|v_{0}^{-}\right|^{p(x)}\right) \mathrm{d} x\right) \\
& \quad-t_{0}^{1-\alpha^{+}}\left(1-\alpha^{+}\right)\left(\lambda \int_{\Omega} a(x)\left|u_{0}^{-}\right|^{1-\alpha(x)} \mathrm{d} x+\mu \int_{\Omega} b(x)\left|v_{0}^{-}\right|^{1-\alpha(x)} \mathrm{d} x\right) \\
& \quad-\left(q^{-}+r^{-}\right) t_{0}^{q^{-}+r^{-}} \int_{\partial \Omega} c(x)\left|u_{0}^{-}\right|^{q(x)}\left|v_{0}^{-}\right|^{r(x)} \mathrm{d} x \\
& \leq\left(t_{0}^{p^{+}} p^{+}-t_{0}^{q^{-}+r^{-}}\left(q^{-}+r^{-}\right)\right) \\
& \quad \times\left(\int_{\Omega}\left(\left|\nabla u_{0}^{-}\right|^{p(x)}+\left|u_{0}^{-}\right|^{p(x)}\right) \mathrm{d} x+\int_{\Omega}\left(\left|\nabla v_{0}^{-}\right|^{p(x)}+\left|v_{0}^{-}\right|^{p(x)}\right) \mathrm{d} x\right) \\
& \quad+\left(t_{0}^{q^{-}+r^{-}}\left(q^{-}+r^{-}\right)-\left(1-\alpha^{+}\right) t_{0}^{1-\alpha^{+}}\right) \\
& \quad \times\left(\lambda \int_{\Omega} a(x)\left|u_{0}^{-}\right|^{1-\alpha(x)} \mathrm{d} x+\mu \int_{\Omega} b(x)\left|v_{0}^{-}\right|^{1-\alpha(x)} \mathrm{d} x\right) \\
& \leq 2\left(t_{0}^{p^{+}} p^{+}-t_{0}^{q^{-}+r^{-}}\left(q^{-}+r^{-}\right)\right)\left\|\left(u_{0}^{-}, v_{0}^{-}\right)\right\|^{p^{-}} \\
& +c_{5}\left(t_{0}^{q^{-}+r^{-}}\left(q^{-}+r^{-}\right)-\left(1-\alpha^{+}\right) t_{0}^{1-\alpha^{+}}\right)(\lambda+\mu)\left\|\left(u_{0}^{-}, v_{0}^{-}\right)\right\|^{1-\alpha^{+}} . \\
& \quad
\end{aligned}
$$

Since $1-\alpha^{+}<p^{+}<q^{-}+r^{-}$, it follows that $I_{\lambda, \mu}^{\prime}\left(t_{0} u_{0}^{-}, t_{0} v_{0}^{-}\right)<0$. Hence, by the definition of $\mathcal{N}_{\lambda, \mu}^{-}$, we have that $\left(t_{0} u_{0}^{-}, t_{0} v_{0}^{-}\right) \in \mathcal{N}_{\lambda, \mu}^{-}$. Now, we shall prove that $u_{n}^{-} \rightarrow u_{0}^{-}$strongly in $W^{1, p(x)}(\Omega)$ and $v_{n}^{-} \rightarrow v_{0}^{-}$strongly in $W^{1, p(x)}(\Omega)$. Suppose 
otherwise; then either

$$
\left\|u_{0}^{-}\right\|_{p} \leq \liminf _{n \rightarrow \infty}\left\|u_{n}^{-}\right\|_{p} \quad \text { or } \quad\left\|v_{0}^{-}\right\|_{p} \leq \liminf _{n \rightarrow \infty}\left\|v_{n}^{-}\right\|_{p} .
$$

Then we have

$$
\begin{aligned}
& E_{\lambda, \mu}\left(t_{0} u_{0}^{-}, t_{0} v_{0}^{-}\right) \leq \frac{t_{0}^{p^{+}}}{p^{-}} L\left(u_{0}^{-}, v_{0}^{-}\right)-\frac{t_{0}^{1-\alpha^{+}}}{1-\alpha^{+}} P\left(u_{0}^{-}, v_{0}^{-}\right)-\frac{t_{0}^{q^{-}+r^{-}}}{q^{+}+r^{+}} Q\left(u_{0}^{-}, v_{0}^{-}\right) \\
& \quad \leq \lim _{n \rightarrow \infty}\left[\frac{t_{0}^{p^{+}}}{p^{-}} L\left(u_{n}^{-}, v_{n}^{-}\right)-\frac{t_{0}^{1-\alpha^{+}}}{1-\alpha^{+}} P\left(u_{n}^{-}, v_{n}^{-}\right)-\frac{t_{0}^{q^{-}+r^{-}}}{q^{+}+r^{+}} Q\left(u_{n}^{-}, v_{n}^{-}\right)\right] \\
& \quad \leq \lim _{n \rightarrow \infty} E_{\lambda, \mu}\left(t_{0} u_{n}^{-}, t_{0} v_{n}^{-}\right) \leq \lim _{n \rightarrow \infty} E_{\lambda, \mu}\left(u_{n}^{-}, v_{n}^{-}\right)=\inf _{(u, v) \in \mathcal{N}_{\lambda, \mu}^{-}} E_{\lambda, \mu}(u, v)=c_{\lambda, \mu}^{-},
\end{aligned}
$$

which contradicts the fact that $\left(t_{0} u_{0}^{-}, t_{0} v_{0}^{-}\right) \in \mathcal{N}_{\lambda, \mu}^{-}$. Thus,

$$
u_{n}^{-} \rightarrow u_{0}^{-} \text {strongly in } W^{1, p(x)}(\Omega)
$$

and

$$
v_{n}^{-} \rightarrow v_{0}^{-} \text {strongly in } W^{1, p(x)}(\Omega) .
$$

This implies that $E_{\lambda, \mu}\left(u_{0}^{-}, v_{0}^{-}\right)=\inf _{(u, v) \in \mathcal{N}_{\lambda, \mu}^{-}} E_{\lambda, \mu}(u, v)$. The proof is now complete.

Proof of Theorem 1.1. To prove Theorem 1.1. let us start by proving the existence of non-negative solutions. First, by Theorems 4.2 and 5.2 we conclude that there exist $\left(u_{0}^{+}, v_{0}^{+}\right) \in \mathcal{N}_{\lambda, \mu}^{+}$and $\left(u_{0}^{-}, v_{0}^{-}\right) \in \mathcal{N}_{\lambda, \mu}^{-}$satisfying

$$
E_{\lambda, \mu}\left(u_{0}^{+}, v_{0}^{+}\right)=\inf _{(u, v) \in \mathcal{N}_{\lambda, \mu}^{+}} E_{\lambda, \mu}(u, v)
$$

and

$$
E_{\lambda, \mu}\left(u_{0}^{-}, v_{0}^{-}\right)=\inf _{(u, v) \in \mathcal{N}_{\lambda, \mu}^{-}} E_{\lambda, \mu}(u, v) .
$$

Moreover, since $E_{\lambda, \mu}\left(u_{0}^{+}, v_{0}^{+}\right)=E_{\lambda, \mu}\left(\left|u_{0}^{+}\right|,\left|v_{0}^{+}\right|\right)$and $\left(\left|u_{0}^{+}\right|,\left|v_{0}^{+}\right|\right) \in \mathcal{N}_{\lambda, \mu}^{+}$, and similarly $E_{\lambda}\left(u_{0}^{-}, v_{0}^{-}\right)=E_{\lambda, \mu}\left(\left|u_{0}^{-}\right|,\left|v_{0}^{-}\right|\right)$and $\left(\left|u_{0}^{-}\right|,\left|v_{0}^{-}\right|\right) \in \mathcal{N}_{\lambda, \mu}^{-}$, we may assume that $\left(u_{0}^{ \pm}, v_{0}^{ \pm}\right) \geq 0$. By Lemma 3.2 . $\left(u_{0}^{ \pm}, v_{0}^{ \pm}\right)$are critical points of $E_{\lambda, \mu}$ on $W$, and hence are weak solutions of problem (1.1). Finally, by the Harnack inequality due to [31], we obtain that $\left(u_{0}^{ \pm}, v_{0}^{ \pm}\right)$are positive solutions of problem (1.1). It remains to show that the solutions found in Theorems 4.2 and 5.2 are distinct. Since $\mathcal{N}_{\lambda, \mu}^{-} \cap \mathcal{N}_{\lambda, \mu}^{+}=\emptyset$, $\left(u_{0}^{ \pm}, v_{0}^{ \pm}\right)$are distinct. The proof is now complete.

\section{ACKNOWLEDGMENTS}

The authors would like to thank the anonymous referees for their carefully reading this paper and several useful comments. 


\section{REFERENCES}

[1] E. Acerbi and G. Mingione, Regularity results for a class of functionals with non-standard growth, Arch. Ration. Mech. Anal. 156 (2001), no. 2, 121-140. MR 1814973

[2] J. Chabrowski and Y. Fu, Existence of solutions for $p(x)$-Laplacian problems on a bounded domain, J. Math. Anal. Appl. 306 (2005), no. 2, 604-618. MR 2136336

[3] M. M. Coclite and G. Palmieri, On a singular nonlinear Dirichlet problem, Comm. Partial Differential Equations 14 (1989), no. 10, 1315-1327. MR 1022988

[4] M. G. Crandall, P. H. Rabinowitz and L. Tartar, On a Dirichlet problem with a singular nonlinearity, Comm. Partial Differential Equations 2 (1977), no. 2, 193-222. MR 0427826

[5] L. Diening, Theoretical and numerical results for electrorheological fluids, Ph.D. thesis, University of Freiburg, Germany, 2002.

[6] P. Drábek and S. I. Pohozaev, Positive solutions for the $p$-Laplacian: application of the fibering method, Proc. Roy. Soc. Edinburgh Sect. A 127 (1997), no. 4, 703-726. MR 1465416

[7] X. Fan and X. Han, Existence and multiplicity of solutions for $p(x)$-Laplacian equations in $\mathbf{R}^{N}$, Nonlinear Anal. 59 (2004), no. 1-2, 173-188. MR 2092084

[8] X. Fan, J. Shen and D. Zhao, Sobolev embedding theorems for spaces $W^{k, p(x)}(\Omega)$, J. Math. Anal. Appl. 262 (2001), no. 2, 749-760. MR 1859337.

[9] X.-L. Fan and Q.-H. Zhang, Existence of solutions for $p(x)$-Laplacian Dirichlet problem, Nonlinear Anal. 52 (2003), no. 8, 1843-1852. MR 1954585

[10] X. Fan, Q. Zhang and D. Zhao, Eigenvalues of $p(x)$-Laplacian Dirichlet problem, J. Math. Anal. Appl. 302 (2005), no. 2, 306-317. MR 2107835.

[11] X. Fan and D. Zhao, On the spaces $L^{p(x)}(\Omega)$ and $W^{m, p(x)}(\Omega)$, J. Math. Anal. Appl. 263 (2001), no. 2, 424-446. MR 1866056

[12] A. Ghanmi and K. Saoudi, The Nehari manifold for a singular elliptic equation involving the fractional Laplace operator, Fract. Differ. Calc. 6 (2016), no. 2, 201-217. MR 3612340

[13] A. Ghanmi and K. Saoudi, A multiplicity results for a singular problem involving the fractional p-Laplacian operator, Complex Var. Elliptic Equ. 61 (2016), no. 9, 1199-1216. MR 3508257

[14] M. Ghergu and V. D. Rădulescu, Singular elliptic problems: bifurcation and asymptotic analysis, Oxford Lecture Series in Mathematics and its Applications, 37, The Clarendon Press, Oxford University Press, Oxford, 2008. MR 2488149

[15] J. Giacomoni and K. Saoudi, Multiplicity of positive solutions for a singular and critical problem, Nonlinear Anal. 71 (2009), no. 9, 4060-4077. MR 2536312

[16] O. Kováčik and J. Rákosník, On spaces $L^{p(x)}$ and $W^{k, p(x)}$, Czechoslovak Math. J. 41(116) (1991), no. 4, 592-618. MR 1134951

[17] J. Liu, Positive solutions of the $p(x)$-Laplace equation with singular nonlinearity, Nonlinear Anal. 72 (2010), no. 12, 4428-4437. MR 2639191

[18] R. A. Mashiyev et al., The Nehari manifold approach for Dirichlet problem involving the $p(x)$-Laplacian equation, J. Korean Math. Soc. 47 (2010), no. 4, 845-860. MR 2667776.

[19] M. Mihăilescu and V. Rădulescu, A multiplicity result for a nonlinear degenerate problem arising in the theory of electrorheological fluids, Proc. R. Soc. Lond. Ser. A Math. Phys. Eng. Sci. 462 (2006), no. 2073, 2625-2641. MR 2253555

[20] S. H. Rasouli, On a PDE involving the variable exponent operator with nonlinear boundary conditions, Mediterr. J. Math. 12 (2015), no. 3, 821-837. MR 3376814

[21] S. H. Rasouli and K. Fallah, On a class of elliptic systems involving the $p(x)$-Laplacian and nonlinear boundary conditions, Izv. Nats. Akad. Nauk Armenii Mat. 49 (2014), no. 4, 47-60; reprinted in J. Contemp. Math. Anal. 49 (2014), no. 4, 175-183. MR 3308633

[22] S. Saiedinezhad and M. B. Ghaemi, The fibering map approach to a quasilinear degenerate $p(x)$-Laplacian equation, Bull. Iranian Math. Soc. 41 (2015), no. 6, 1477-1492.|MR 3437159 
[23] K. Saoudi, The fibering map approach to a $p(x)$-Laplacian equation with singular nonlinearities and nonlinear Neumann boundary conditions, Rocky Mountain J. Math. 48 (2018), no. 3, 927-946. MR 3835580

[24] K. Saoudi, Existence and non-existence of solutions for a singular problem with variable potentials, Electron. J. Differential Equations 2017, Paper No. 291, 9 pp. MR 3748009

[25] K. Saoudi, Existence and multiplicity of solutions for a quasilinear equation involving the p(x)-Laplace operator, Complex Var. Elliptic Equ. 62 (2017), no. 3, 318-332. MR 3598980

[26] K. Saoudi, Existence and non-existence of solution for a singular nonlinear Dirichlet problem involving the $\mathrm{p}(x)$-Laplace operator, J. Adv. Math. Stud. 9 (2016), no. 2, 291-302. MR 3559394

[27] K. Saoudi and A. Ghanmi, A multiplicity results for a singular equation involving the $p(x)$ Laplace operator, Complex Var. Elliptic Equ. 62 (2017), no. 5, 695-725. MR 3613680.

[28] K. Saoudi and M. Kratou, Existence of multiple solutions for a singular and quasilinear equation, Complex Var. Elliptic Equ. 60 (2015), no. 7, 893-925. MR 3350472

[29] K. Saoudi, M. Kratou and S. Alsadhan, Multiplicity results for the $p(x)$-Laplacian equation with singular nonlinearities and nonlinear Neumann boundary condition, Int. J. Differ. Equ. 2016, Art. ID 3149482, 14 pp. MR 3574266.

[30] A. Taghavi, G. A. Afrouzi and H. Ghorbani, The Nehari manifold approach for $p(x)$-Laplacian problem with Neumann boundary condition, Electron. J. Qual. Theory Differ. Equ. 2013, No. 39, 14 pp. MR 3081562

[31] X. Zhang and X. Liu, The local boundedness and Harnack inequality of $p(x)$-Laplace equation, J. Math. Anal. Appl. 332 (2007), no. 1, 209-218. MR 2319655

\section{Mouna Kratou}

College of Sciences at Dammam, Imam Abdulrahman Bin Faisal University, 31441 Dammam, Kingdom of Saudi Arabia

Basic and Applied Scientific Research Center, Imam Abdulrahman Bin Faisal University,

P.O. Box 1982, 31441 Dammam, Kingdom of Saudi Arabia

mmkratou@iau.edu.sa

Kamel Saoudi ${ }^{凶}$

College of Sciences at Dammam, Imam Abdulrahman Bin Faisal University, 31441 Dammam, Kingdom of Saudi Arabia

Basic and Applied Scientific Research Center, Imam Abdulrahman Bin Faisal University,

P.O. Box 1982, 31441 Dammam, Kingdom of Saudi Arabia

kmsaoudi@iau.edu.sa

Received: October 21, 2018

Accepted: September 15, 2019 